\title{
Observation of sub-natural linewidths for cold atoms in a magneto-optic trap
}

\author{
Umakant D. Rapol, Ajay Wasan, and Vasant Natarajan* \\ Department of Physics, Indian Institute of Science, Bangalore 560 012, INDIA
}

\begin{abstract}
We have studied the absorption of a weak probe beam through cold rubidium atoms in a magneto-optic trap. The absorption spectrum shows two peaks with the smaller peak having linewidth as small as $28 \%$ of the natural linewidth. The modification happens because the laser beams used for trapping also drive the atoms coherently between the ground and excited states. This creates "dressed" states whose energies are shifted depending on the strength of the drive. Linewidth narrowing occurs due to quantum coherence between the dressed states. The separation of the states increases with laser intensity and detuning, as expected from this model.
\end{abstract}

Typeset using REVTEX

*E-mail: vasant@physics.iisc.ernet.in 
The use of near-resonant laser light to cool atoms from room temperature to ultra-low temperatures has become a standard technique for atomic physics experiments [1]. Temperatures in the range of few microkelvin are routinely achieved using these methods. The cooled atoms can also be trapped by superposing a magnetic field on the laser beams in a configuration called a magneto-optic trap (MOT) [2]. Cold atoms in a MOT form near-ideal laboratories to test our understanding of the fundamental laws of physics. There are two primary reasons for this. The first is that almost all laser cooling experiments are done on alkali atoms, and alkali atoms are attractive candidates for such fundamental experiments because their simple one-electron structure renders them amenable to theoretical calculations. For example, the alkali atom cesium has been used for high-precision experiments on atomic parity violation [3] because the experimental results can be compared to theoretical predictions based on the Standard Model. The second advantage of cold atoms in a MOT is that, if one is looking for spectroscopic signatures of some effect, then the use of ultra-cold atoms guarantees high precision because Doppler broadening is negligible. For example, a probe beam sent through a cloud of rubidium atoms in a MOT shows a Doppler width of 500 $\mathrm{kHz}$, while the natural linewidth of the cooling transition is $6.1 \mathrm{MHz}$. However, the natural linewidth appears as a fundamental limit to the resolution achievable in these experiments. In addition, it is often necessary to turn off the MOT before doing any precision measurement because the trapping beams cause unwanted perturbation to the energy levels of the atoms. In this article, we show that, not only can the energy shift from the trapping beams be well understood from a simple theoretical model, but this can also lead to significant narrowing of the linewidth of the transition below the natural linewidth.

The MOT was first invented in 1987 [2] and since then it has become the workhorse for any experiment that requires a cold and dense cloud of atoms. It is the starting point for experiments ranging from atomic fountain clocks to achieving Bose-Einstein condensation «4 4 . We have been interested in a MOT primarily from its potential use in precision spectroscopy experiments. The standard MOT configuration consists of three pairs of counter-propagating laser beams in the three orthogonal directions, and a quadrupole magnetic field generated 
using two coils carrying current in opposite directions. The laser beams are circularly polarized and detuned below the resonance by 2 to $3 \Gamma$, where $\Gamma$ is the natural linewidth of the cooling transition.

We work with ${ }^{87} \mathrm{Rb}$ atoms in the MOT, for which the cooling transition is the $5 S_{1 / 2}, F=$ $2 \leftrightarrow 5 P_{3 / 2}, F^{\prime}=3$ transition at $780 \mathrm{~nm}$. The transition has a natural linewidth of $6.1 \mathrm{MHz}$ and saturation intensity [6] of $1.6 \mathrm{~mW} / \mathrm{cm}^{2}$. The laser beams are derived from a frequencystabilized diode laser system locked near this transition. To prevent the atoms from optically pumping out of the cooling transition, a small amount of "repumping" light resonant with the $5 S_{1 / 2}, F=1 \leftrightarrow 5 P_{3 / 2}, F^{\prime}=2$ transition is mixed with the primary MOT beams. The repumping beam is obtained from a second tunable diode laser system. The experiments are done in a vacuum chamber maintained at a pressure below $10^{-9}$ torr. Rb atoms are loaded into the trap using a getter source [7] that fills the chamber with Rb vapor when heated with a few amps of current. The low-energy tail from this background vapor is cooled and captured in the MOT.

The loading of atoms into the MOT follows an exponential growth curve [7]. The time constant is a few seconds, so that, after about a minute, the MOT is in steady state with a number of atoms that stays constant as long as the source remains on. Under typical conditions, the steady-state MOT has about $10^{8}$ atoms at a temperature of $300 \mu \mathrm{K}$ and density of $10^{10}$ atoms/cc. We have studied the absorption of a weak probe beam passing through the cold atoms in a MOT after it has attained steady state. The probe is linearly polarized and has an intensity of about $250 \mu \mathrm{W} / \mathrm{cm}^{2}$. A typical absorption spectrum as the probe laser is scanned across the $5 S_{1 / 2}, F=2 \leftrightarrow 5 P_{3 / 2}, F^{\prime}=3$ transition is shown in Fig. 1. The main features of the spectrum are that there are two peaks instead of one, and that the linewidth of the smaller peak is only $1.7 \mathrm{MHz}$, or about $28 \%$ of the natural linewidth.

To understand this we have to consider that, in the presence of the probe and trapping beams, the Rb atoms form an effective three-level system as shown in Fig. 2. In other words, the circularly-polarized trapping beam couples certain magnetic sublevels in the ground and excited state, while the linearly-polarized probe beam measures absorption from the 
same ground-state sublevel to a different excited-state sublevel. For the trapping beam, we only consider the stretched state, i.e. the magnetic sublevel where the projection of angular momentum $\left(m_{F}\right)$ is maximum. The main reason for this is that, as the atoms move away from the trap center, they rapidly get optically pumped into this state. Once they are in this state they remain there until they go to the other side of the trap where they get optically pumped into the opposite stretched state. For example, the $\sigma^{+}$beam optically pumps atoms into the $5 S_{1 / 2}, F=2, m_{F}=2$ sublevel from where they cycle on the $F=2, m_{F}=2 \leftrightarrow F^{\prime}=3, m_{F^{\prime}}=3$ transition, while on the other side of the trap, the $\sigma^{-}$ beam optically pumps atoms into the $5 S_{1 / 2}, F=2, m_{F}=-2$ sublevel.

The primary purpose of the trapping beam is to provide a radiation pressure force that pushes the atoms towards the trap center. This force arises because the atom absorbs a laser photon, goes to the excited state, and then decays to the ground state through spontaneous emission. However, there is a small probability that the atom decays through stimulated emission induced by the same beam. The resulting force is called the stimulated force and causes the atom to be coherently driven between the ground and excited states. The frequency at which the probability swaps between the two states is called the Rabi frequency, $\Omega_{R}$, and is determined by the laser intensity and the strength of the transition [8]. Since we are going to be interested only in this coherent driving of the atom, this gives another justification for considering only the stretched state in our analysis: transitions starting from this state are the strongest in terms of relative oscillator strength.

The energy levels shown in Fig. 2 can now be understood more clearly. The $\sigma^{+}$trapping beam couples the $5 S_{1 / 2}, F=2, m_{F}=2$ and $5 P_{3 / 2}, F^{\prime}=3, m_{F^{\prime}}=3$ levels, while the probe measures absorption on the $5 S_{1 / 2}, F=2, m_{F}=2 \rightarrow 5 P_{3 / 2}, F^{\prime}=3, m_{F^{\prime}}=2$ transition. The trapping beam has a detuning from resonance of $\Delta_{t}$ and the probe beam has a detuning of $\Delta$. The spontaneous decay rates from the excited levels, $\Gamma_{21}$ and $\Gamma_{31}$, are both equal to $\Gamma$, which is $6.1 \mathrm{MHz}$. As discussed above, the trapping beam partly drives the atoms coherently. It is well known that this driving creates "dressed" states whose energies are shifted by the strength of the drive (also called the ac Stark shift) [9]. The energy shift is 
analogous to the shift in the frequency of two classical oscillators that are coupled together, where the shift is proportional to the strength of the coupling. In fact, this analogy with coupled classical oscillators can be made more exact, and results in analogous definitions of classical dressed states and Rabi frequency [10].

In the presence of the dressed states, the probe-absorption spectrum splits into two peaks, called an Autler-Townes doublet [11. The location of the two peaks is given by [12,13]:

$$
\Delta_{ \pm}=\frac{\Delta_{t}}{2} \pm \frac{1}{2} \sqrt{\Delta_{t}^{2}+\Omega_{R}^{2}}
$$

where $\Delta_{+}$and $\Delta_{-}$are the values of the probe detuning where the peaks occur. The corresponding linewidths $\left(\Gamma_{ \pm}\right)$of these peaks are different because of the coherence between the two dressed states, and given by

$$
\Gamma_{ \pm}=\frac{\Gamma}{2}\left(1 \mp \frac{\Delta_{t}}{\sqrt{\Delta_{t}^{2}+\Omega_{R}^{2}}}\right) .
$$

It is clear from the above expression that, if $\Delta_{t}=0$, the two peaks are symmetric and have identical linewidths of $\Gamma / 2$. However, for any non-zero detuning, the peaks have asymmetric linewidths. The first peak has larger linewidth while the second peak has smaller linewidth by precisely the same factor, in such a way that the sum of the two linewidths is equal to the unperturbed linewidth, $\Gamma$.

The above analysis is for a stationary atom. If the atom is moving, the laser detuning as seen by the atom depends on its velocity. To obtain the probe absorption in a gas of moving atoms, the above expressions have to be corrected for the velocity of the atom and then averaged over the Maxwell-Boltzmann distribution of velocities. Such an analysis has been done by Vemuri et al. in Ref. [13]. The important conclusion of that work is that the location of the peaks given in Eq. 11 does not change, but the linewidths are now given by

$$
\Gamma_{ \pm}=\frac{\Gamma+D}{2}\left(1 \mp \frac{\Delta_{t}}{\sqrt{\Delta_{t}^{2}+\Omega_{R}^{2}}}\right) .
$$

Here, $D$ is the Doppler width, which is $0.5 \mathrm{MHz}$ for Rb atoms at a temperature of 300 $\mu \mathrm{K}$. This is negligible compared to the natural linewidth of $6.1 \mathrm{MHz}$. Therefore, for our conditions, Eqs. 1 and 2 can be taken as valid even at the finite temperature in the trap. 
The probe-absorption spectrum presented in Fig. 1 1 can now be understood based on the above analysis. The trapping beam coherently drives the atoms and creates two dressed states near the original ground state. The probe beam measures absorption from the modified ground state and therefore shows two peaks. For non-zero detuning of the trapping beam, the linewidth of the smaller peak is much smaller than $\Gamma$, as given in Eq. 2 .

In order to verify that this model is correct, we have studied the probe absorption at different values of trapping-beam intensity (or Rabi frequency) and detuning. From Eq. 1, the separation of the two peaks in the absorption spectrum is $\sqrt{\Delta_{t}^{2}+\Omega_{R}^{2}}$, and should increase when either $\Omega_{R}$ or $\Delta_{t}$ is increased. The variation with laser intensity at three different values of $\Delta_{t}$ is shown in Fig. [3. The solid line is a fit to the variation expected from Eq. 1 and matches the data quite well. However, for each curve, the $y$-intercept should be the detuning $\Delta_{t}$. Instead, we find that the intercept is smaller by about $4 \mathrm{MHz}$. There could be several reasons for this. One likely explanation is that, because the atoms are in a linearly-varying magnetic field, the detuning of the trapping laser depends on the exact location of the atom. Atoms that get optically pumped into the stretched state are most likely to be near the periphery of the cloud. Taking into account the $4 \mathrm{~mm}$ size of the cloud and the magnetic-field gradient of $10 \mathrm{G} / \mathrm{cm}$, the field at this location is about $2 \mathrm{G}$. For the stretched state, this would cause a decrease in detuning by $2.8 \mathrm{MHz}$, which is about the size of the decrease we measure. In addition, atoms away from the center of the trap have a non-zero velocity. Because the lasers are detuned below resonance, optical pumping into the stretched state is most likely when the atoms are moving towards the laser beam. The Doppler shift in this situation is such that it would again cause a reduction in the detuning. For atoms moving with the r.m.s. velocity in the trap, the Doppler shift is about $0.4 \mathrm{MHz}$.

Whatever be the exact cause for this change in detuning seen by the atoms, we can verify its effect further by studying the separation of the peaks as a function of detuning. In Fig. 因, we show the separation $v$ s. detuning for different values of trapping-beam intensity. The solid lines are exact calculations from Eq. 1 and the measured points lie almost perfectly on these curves. However, to get this match, we had to use the effective detuning from the 
$y$-intercepts of Fig. 3, and not the zero-field detuning set in the laboratory. The excellent agreement with the theoretical prediction gives us confidence that the atoms are indeed seeing a smaller detuning.

There are other features of the absorption spectra that are clearly noticeable in the data. For example, the smaller peak becomes more prominent (relative to the larger peak) as the trapping-beam intensity is increased at a given detuning. Similarly, for a fixed intensity, the peak becomes more prominent at smaller detunings. Both these observations are consistent with the model of a driven two-level system, since the effect of the drive is stronger when the intensity is higher or when it is closer to resonance. However, there are some complications with this simple model that are also evident in the data. One problem is that the linearlypolarized probe laser can also couple to the $F^{\prime}=3, m_{F^{\prime}}=1$ sublevel, in addition to the $F^{\prime}=3, m_{F^{\prime}}=2$ sublevel already considered. The location of the $F^{\prime}=3, m_{F^{\prime}}=1$ absorption peak is slightly shifted due to the non-zero magnetic field. This transition is very weak in terms of relative strength compared to the transition to the $F^{\prime}=3, m_{F^{\prime}}=2$ level, therefore it would show up only in the primary absorption peak. But this implies that the primary peak is a convolution of two peaks. We see some evidence for this in the data because we find that the lineshape of the primary peak is non-Lorentzian. This makes it difficult to extract a reliable estimate of the linewidth of the peak but does not affect the location of the peak center that has been used for Figs. 3 and 4 .

In conclusion, we have observed sub-natural linewidth for probe absorption through cold rubidium atoms in a MOT. The modification to the absorption spectrum arises because of dressed states created by the trapping beams. Perhaps the most useful way to understand this is in the context of driven three-level systems [14,12] and the phenomenon of electromagnetically induced transparency (EIT) [15]. In EIT, an initially absorbing medium is made transparent to the probe when a strong control laser is applied to an auxiliary transition. In our case, the trapping laser itself plays the role of the control laser that modifies the absorption of the probe. It thus opens up new possibilities for EIT experiments using cold atoms, where phenomena such as gain without inversion, anomalous dispersion, and 
population trapping can be studied.

\section{ACKNOWLEDGMENTS}

This work was supported by a research grant from the Department of Science and Technology, Government of India. 


\section{REFERENCES}

[1] A good review of laser cooling and trapping experiments is contained in the Nobel Prize lectures: S. Chu, Rev. Mod. Phys. 70, 685 (1998), C. N. Cohen-Tannoudji, ibid. 70, 707 (1998); and W. D. Phillips, ibid. 70, 721 (1998).

[2] E. L. Raab et al., Phys. Rev. Lett. 59, 2631 (1987).

[3] C. S. Wood et al., Science 275, 1759 (1997).

[4] M. H. Anderson et al., Science 269, 198 (1995).

[5] For a recent review, see W. Ketterle, Phys. Today 52, 30 (1999).

[6] The saturation intensity is a measure of the strength of the laser-induced coupling between the ground and excited states. It is defined as that intensity for which the transition is power broadened by a factor of $\sqrt{2}$.

[7] U. D. Rapol, A. Wasan, and V. Natarajan, Phys. Rev. A 64, 023402 (2001).

[8] The relation between the intensity and the Rabi frequency is $I / I_{0}=2 \Omega_{R}^{2} / \Gamma^{2}$, where $I_{0}$ is the saturation intensity.

[9] C. Cohen-Tannoudji and S. Reynaud, J. Phys. B. 10, 365 (1977).

[10] E. A. Cornell, R. M. Weisskoff, K. R. Boyce, and D. E. Pritchard, Phys. Rev. A 41, $312(1990)$.

[11] S. H. Autler and C. H. Townes, Phys. Rev. 100, 703 (1955).

[12] G. S. Agarwal, Phys. Rev. A. 54, R3734 (1996).

[13] G. Vemuri, G. S. Agarwal, and B. D. Nageswara Rao, Phys. Rev. A. 53, 2842 (1996).

[14] L. M. Narducci et al., Phys. Rev. A. 42, 1630 (1990).

[15] See, for example, S. E. Harris, Phys. Today 50, 36 (1997). 


\section{FIGURES}

FIG. 1. The figure shows the absorption of the probe beam as a function of its detuning from the $5 S_{1 / 2}, F=2 \leftrightarrow 5 P_{3 / 2}, F^{\prime}=3$ transition. The important feature is the appearance of two peaks instead of one. The dotted line is a Lorentzian fit to the two peaks and yields linewidths (FWHM) of $14 \mathrm{MHz}$ for the larger peak and only $1.7 \mathrm{MHz}(0.28 \Gamma)$ for the smaller peak. The trapping beam detuning $\Delta_{t}$ is $-19 \mathrm{MHz}$, and the intensity $I$ is $4.8 \mathrm{~mW} / \mathrm{cm}^{2}$.

FIG. 2. Effective three-level system in ${ }^{87} \mathrm{Rb}$ when both trapping and probe beams are on. The trapping laser is circularly polarized and optically pumps the atoms into the stretched state. For $\sigma^{+}$-pol, the trapping laser couples the $F=2, m_{F}=2$ and $F^{\prime}=3, m_{F^{\prime}}=3$ levels at a detuning $\Delta_{t}$. The probe laser is linearly polarized and measures absorption on the $F=2, m_{F}=2 \rightarrow F^{\prime}=3, m_{F^{\prime}}=2$ transition at a detuning $\Delta . \Gamma_{31}$ and $\Gamma_{21}$ are the spontaneous decay rates.

FIG. 3. The figure shows the separation of the two absorption peaks as a function of trapping-beam intensity, for three values of detuning. The solid lines are fits to Eq. 1 in the text. From Eq. 1 , the $y$-intercept in each case must be $\Delta_{t}$, but the value from the fit is lower by about $4 \mathrm{MHz}$. This indicates that the effective detuning seen by the atoms is smaller. Otherwise, the separation follows the predicted variation very closely.

FIG. 4. The figure shows the separation of the two absorption peaks as a function of detuning, for three values of trapping-beam intensity. The solid lines are solutions to Eq. 1 in the text with no adjustable parameters, provided we use the effective detuning values obtained from the $y$-intercepts in Fig. 3 and not the values we set. Note the excellent agreement with theory under this assumption. 


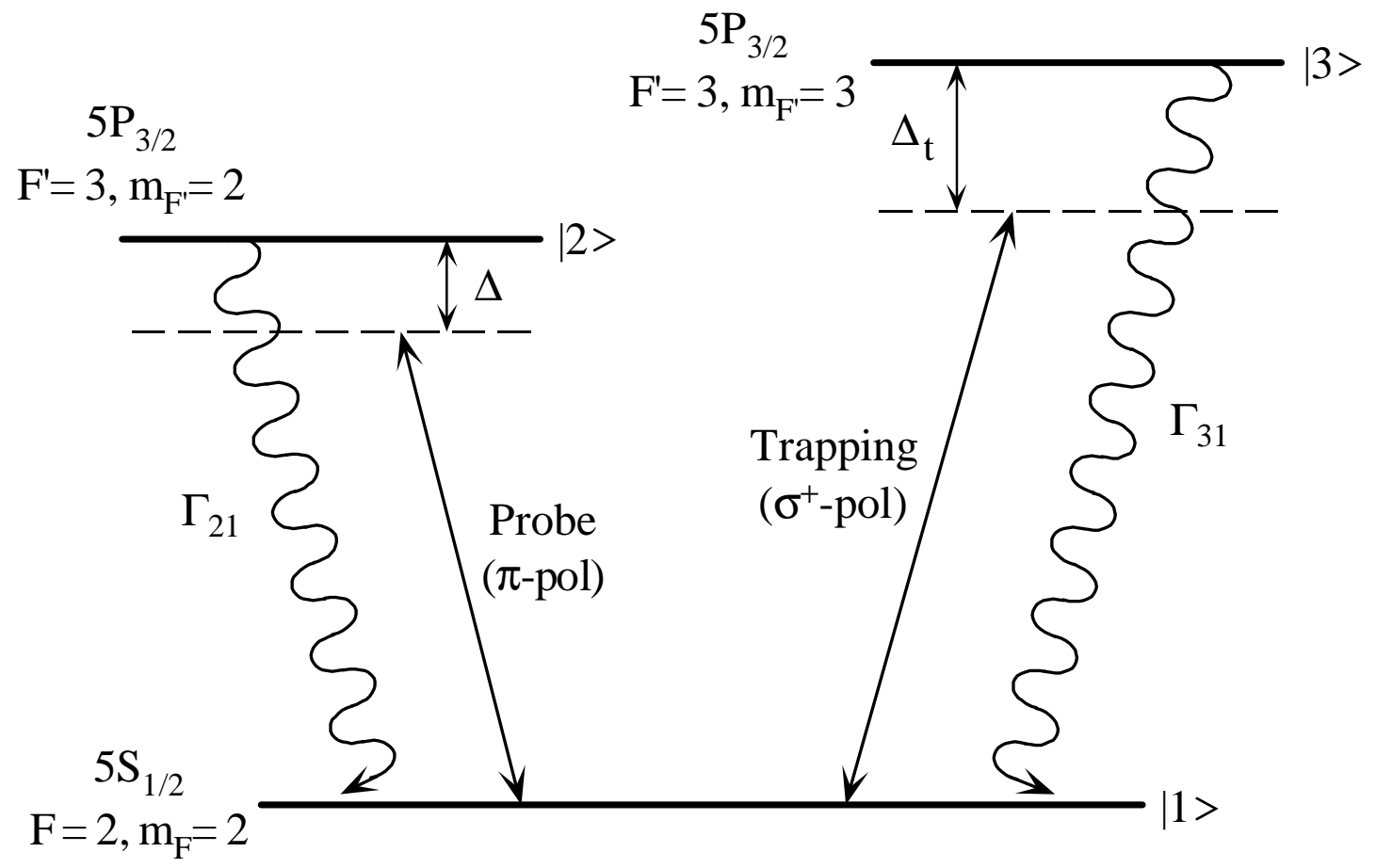

